\title{
One Pot Synthesis of Bioactive Novel Cyanopyridones
}

\author{
Kamlesh Khokhani, Taslimahemad Khatri ${ }^{\dagger+*}$, and Praful Patel \\ Department of Chemistry, M M Science College, Morbi-363 642, Gujarat, India \\ ${ }^{\dagger}$ Department of Chemistry, KSKV Kachchh University, Bhuj-Kachchh 370 001, Gujarat, India \\ *E-mail: Khatri_taslim@yahoo.com
}

(Received April 9, 2013; Accepted July 15, 2013)

\begin{abstract}
Cyanopyridone was prepared by the condensation of cyanoacetamide, substituted arylaldehydes and malononitrile in presence of pipyridine. The structure of the synthesized compound CP 1-20 was assigned on the basis of elemental analysis, IR, ${ }^{1} \mathrm{H}-\mathrm{NMR}$ and mass spectroscopy. These compounds were also screened for antimicrobial activity. The Minimum Inhibitory Concentration (MIC) of all the synthesized compounds was compared with standard drugs.
\end{abstract}

Key words: Cyanopyrimidones, Cyanoacetamide, Antimicrobial activity, Malononitrile, Pipyridine

\section{INTRODUCTION}

Pyridone and their derivatives play an essential role in several biological processes and have considerable chemical and pharmacological importance. ${ }^{1-3}$ The 2-pyridones represent a unique class of pharmacophore, which are observed in various therapeutic agents ${ }^{4}$ and antibiotics. ${ }^{5}$ The 3-cyanopyridin-2-one nucleus is the structural basis of the alkaloid ricinine (I), the first known alkaloid containing a cyano-group. Cheney et al. reported 4,6-diaryl2-oxo-1,2-dihydropyridine-3-carbonitriles (II), as inhibitors of the oncogenic serine/threonine kinas PIM-1, which plays a role in cancer cell survival, differentiation and proliferation. ${ }^{6}$ Wendt et al. showed that several compounds with the same general formula as above (II) but with higher lipophilic properties (III) can inhibit surviving which is a member of the inhibitor of apoptosis family (IAP). ${ }^{7}$

The thienopyridone agonist (IV) showed modest AMPK (adenosine monophosphate-activated protein kinase) activity $^{8}$ (Fig. 1). These heterocycles attracted attention because of their applications as bioactive compounds for example as a promising class of HIV-1 non-nucleoside reverse transcriptase inhibitors (NNRTIs) ${ }^{9}$ as antibacterial, ${ }^{10}$ antifungal, ${ }^{11}$ sedative, ${ }^{12}$ andcardiotonic agent. ${ }^{13}$ Moreover, such derivatives have recently become important due to their structural similarity to nucleosides. ${ }^{14}$

They are also versatile precursors for the construction of complex natural products ${ }^{15}$ pyridines ${ }^{16}$ and larger pyridone systems such as those found in the nitro guanidine insecticide Imidacloprid ${ }^{17}$ and subtype selective GABA receptor agonists. Consequently, methodologies for the prepara-<smiles>COc1ccn(C)c(=O)c1C#N</smiles>

(I) Ricinine<smiles>N#Cc1c(-c2cc(Cl)ccc2Cl)cc(-c2ccccc2)[nH]c1=O</smiles>

(III) Survivin inhibition<smiles>N#Cc1c(-c2ccccc2)cc(-c2cc(Br)ccc2O)[nH]c1=O</smiles>

(II) PIM-1 kinase inhibition<smiles>N#Cc1c(O)c2c(-c3ccccc3)csc2[nH]c1=O</smiles>

(IV) AMPK Activator
Figure 1. Some reported potent cynopyridones.

tion of pyridones have attracted much attention from both industrial and academic areas.

\section{EXPERIMENTAL}

Melting points were determined in open capillary tubes and are uncorrected. Formation of the compounds was routinely checked by TLC on silica gel-G plates of $0.5 \mathrm{~mm}$ thickness and spots were located by iodine. IR spectra were recorded Shimadzu FT-IR-8400 instrument using $\mathrm{KBr}$ pellet method. Mass spectra were recorded on Shimadzu GC-MS-QP-2010 model using Direct Injection Probe 
<smiles>[R]c1ccc([R])c(N)c1</smiles><smiles>[R2]c1ccc([R])c(NC(=O)CC#N)c1</smiles>

Scheme 1. Synthesis of 2-cyano-N-(substituted) acetamides.

technique. ${ }^{1} \mathrm{H}-\mathrm{NMR}$ was determined using DMSO- $d_{6}$ as asolvent on a Bruker Ac $400 \mathrm{MHz}$ spectrometer. Elemental analysis of the all the synthesized compounds was carried out on Elemental Vario EL III Carlo Erba 1108 model and the results are in agreements with the structures assigned.

\section{Preparations of Cyanoacetamide Derivatives}

The disubstituted aniline $(10 \mathrm{mmol})$ and ethyl 2-cyanoacetate $(10 \mathrm{mmol})$ were refluxed on sand bath for 3-4 hours to yield 2-cyano-N-(substituted) acetamides ${ }^{18}(3)$ in good yields (Scheme 1).

\section{General Procedure for Synthesis of Cyanopyridones (CP 1-20)}

A mixture of substituted $10 \mathrm{mmol}$ of cyanoacetamide (3), $10 \mathrm{mmol}$ substituted aldehyde (4) and $10 \mathrm{mmol}$ of malononitrile (5) were dissolve in $30 \mathrm{ml}$ of methanol, catalytic amount of pipyridine was added. The reaction mixture was heated under reflux on water bath for $20-22 \mathrm{~h}$ (under TLC analysis). After completion of the reaction, filtered product was washed with methanol and recrystalized from ethanol (Scheme 2).

6-Amino-1-(2,5-dichlorophenyl)-2-oxo-4-phenyl-1,2dihydro pyridine-3,5-dicarbonitrile (CP-1):

MP: $272-274^{\circ} \mathrm{C} ;{ }^{1} \mathrm{H}-\mathrm{NMR}\left(400 \mathrm{MHz}, \mathrm{DMSO}-d_{6}\right) \delta \mathrm{ppm}$ :
(3)<smiles>[R3]c1cccc(C=O)c1</smiles>

(4)

(5)
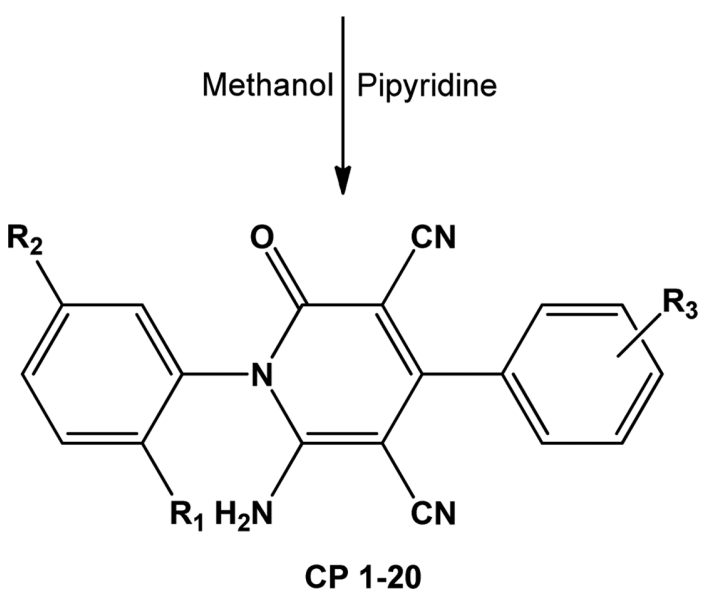

Scheme 2. One spot synthesis of CP 1-20.

7.01-7.11 (t, 1H, Ar-H), 7.10-7.12 (t, 2H, Ar-H, $J=11.6$ $\mathrm{Hz}$ ), 7.52-7.55 (t, 2H, Ar-H, $J=11.6 \mathrm{~Hz}), 7.68-7.69$ (d, 1H, Ar-diCl), 7.75-7.78 (d, 1H, Ar-diCl), 7.85 (s, 1H, $\mathrm{Ar}-\mathrm{diCl}), 8.20$ (s, $\left.2 \mathrm{H}, \mathrm{NH}_{2}\right)$; IR $\left(\mathrm{KBr}, \mathrm{v} / \mathrm{cm}^{-1}\right): 3665$ and $3522\left(\mathrm{NH}_{2}\right), 3194(\mathrm{Ar}-\mathrm{H}), 2218(\mathrm{C} \equiv \mathrm{N}), 1639(\mathrm{C}=\mathrm{O}), 774$ (C-Cl); MS m/z (\%): $380\left(\mathrm{M}^{+}\right), 364(100), 329(6), 304$ (14), 288 (31), 210 (16), 187 (23), 152 (8), 77 (32). Elemental Analysis for $\mathrm{C}_{19} \mathrm{H}_{10} \mathrm{Cl}_{2} \mathrm{~N}_{4} \mathrm{O}$ : Calculated: $\mathrm{C}, 59.86 ; \mathrm{H}, 2.64$; N, 14.70. Found: C, 59.40; H, 2.30; N, 14.35\%.

6-Amino-1-(2,5-dichlorophenyl)-4-(4-methoxyphenyl)2-oxo-1,2-dihydro pyridine-3,5-dicarbonitrile (CP-2): MP: $280-284{ }^{\circ} \mathrm{C} ;{ }^{1} \mathrm{H}-\mathrm{NMR}\left(400 \mathrm{MHz}\right.$, DMSO- $\left.d_{6}\right) \delta \mathrm{ppm}$ : 3.85 (s, $\left.3 \mathrm{H}, \mathrm{OCH}_{3}\right), 7.12-7.15$ (d, $2 \mathrm{H}, 4-\mathrm{OCH}_{3}-\mathrm{Ar}-\mathrm{H}, J$ $=11.6 \mathrm{~Hz}), 7.52-7.55\left(\mathrm{~d}, 2 \mathrm{H}, 4-\mathrm{OCH}_{3}-\mathrm{Ar}-\mathrm{H}, J=11.6 \mathrm{~Hz}\right)$, 7.68-7.69 (d, 1H, diCl-Ar-H), 7.75-7.78 (d, 1H, diClAr-H), 7.85 (s, 1H, diCl-Ar-H), 8.28 (s, 2H, NH $\mathrm{N}_{2}$; IR $\left(\mathrm{KBr}, \mathrm{v} / \mathrm{cm}^{-1}\right)$ : 3665 and $3522\left(\mathrm{NH}_{2}\right), 3194(\mathrm{Ar}-\mathrm{H}), 2218$ $(\mathrm{C} \equiv \mathrm{N}), 1639(\mathrm{C}=\mathrm{O}), 1173(\mathrm{C}-\mathrm{O}-\mathrm{C}), 774(\mathrm{C}-\mathrm{Cl}) ; \mathrm{MS} \mathrm{m} / \mathrm{z}$ (\%): $410\left(\mathrm{M}^{+}\right), 394$ (16), 375 (100), 360 (4), 332 (6), 196 (4), 187 (16), 152 (8), 109 (7), 75 (4). Elemental Analysis for $\mathrm{C}_{20} \mathrm{H}_{12} \mathrm{Cl}_{2} \mathrm{~N}_{4} \mathrm{O}_{2}$ : Calculated: $\mathrm{C}, 58.41 ; \mathrm{H}, 2.94 ; \mathrm{N}, 13.62$. Found: C, 58.21; H, 2.45; N, 13.33\%.

6-Amino-1-(2,5-dichlorophenyl)-4-(2-methoxyphenyl)2-oxo-1,2-dihydro pyridine-3,5-dicarbonitrile (CP-3): MP: $280-284{ }^{\circ} \mathrm{C} ;{ }^{1} \mathrm{H}-\mathrm{NMR}\left(400 \mathrm{MHz}, \mathrm{DMSO}-d_{6}\right) \delta$ 
ppm: 3.87 (s, 3H, $\left.\mathrm{OCH}_{3}\right), 7.10-7.13\left(\mathrm{~d}, 2 \mathrm{H}, 4-\mathrm{OCH}_{3}-\mathrm{Ar}-\mathrm{H}\right.$, $J=11.6 \mathrm{~Hz}), 7.54-7.57\left(\mathrm{~d}, 2 \mathrm{H}, 4-\mathrm{OCH}_{3}-\mathrm{Ar}-\mathrm{H}, J=11.6 \mathrm{~Hz}\right)$, 7.22-7.28 (t, 2H, 4-OCH $-\mathrm{Ar}-\mathrm{H},), 7.65-7.67(\mathrm{~d}, 1 \mathrm{H}$, diCl-Ar-H), 7.71-7.74 (d, 1H, diCl-Ar-H), 7.84 (s, 1H, diCl-Ar-H), 8.28 (s, 2H, NH $\mathrm{N}_{2}$; IR ( $\left.\mathrm{KBr}, \mathrm{v} / \mathrm{cm}^{-1}\right): 3665$ and $3522\left(\mathrm{NH}_{2}\right), 3194(\mathrm{Ar}-\mathrm{C}-\mathrm{H}), 2218(\mathrm{C} \equiv \mathrm{N}), 1639$ $(\mathrm{C}=\mathrm{O}), 1173(\mathrm{C}-\mathrm{O}-\mathrm{C}), 774(\mathrm{C}-\mathrm{Cl}) ; \mathrm{MS} \mathrm{m} / \mathrm{z}(\%): 410\left(\mathrm{M}^{+}\right)$, 394(45), 375(100), 360 (6), 332 (9), 196(8), 187 (21), 152 (6), 109 (11), 75 (8). Elemental Analysis for $\mathrm{C}_{20} \mathrm{H}_{12} \mathrm{Cl}_{2} \mathrm{~N}_{4} \mathrm{O}_{2}$ : Calculated: C, 58.41; H, 2.94; N, 13.62. Found: C, 58.26; $\mathrm{H}, 2.54 ; \mathrm{N}, 13.46 \%$.

6-Amino-1-(2,5-dichlorophenyl)-4-(4-nitrophenyl)-2oxo-1,2-dihydro pyridine-3,5-dicarbonitrile (CP-4):

MP: $245-250^{\circ} \mathrm{C}$; ${ }^{1} \mathrm{H}-\mathrm{NMR}\left(400 \mathrm{MHz}, \mathrm{DMSO}-d_{6}\right) \delta \mathrm{ppm}$ : $8.12-8.15\left(\mathrm{~d}, 2 \mathrm{H}, 4-\mathrm{NO}_{2}-\mathrm{Ar}-\mathrm{H}, J=11.6 \mathrm{~Hz}\right), 7.52-7.55$ (d, $\left.2 \mathrm{H}, 4-\mathrm{NO}_{2}-\mathrm{Ar}-\mathrm{H}, J=11.6 \mathrm{~Hz}\right), 7.68-7.69$ (d, $1 \mathrm{H}, \mathrm{diCl}-$ $\mathrm{Ar}-\mathrm{H}), 7.75-7.78$ (d, 1H, diCl-Ar-H), 7.85 (s, 1H, diCl-Ar$\mathrm{H}), 8.38\left(\mathrm{~s}, 2 \mathrm{H}, \mathrm{NH}_{2}\right)$; IR $\left(\mathrm{KBr}, \mathrm{v} / \mathrm{cm}^{-1}\right): 3665$ and 3522 $\left(\mathrm{NH}_{2}\right), 3194(\mathrm{Ar}-\mathrm{C}-\mathrm{H}), 2218(\mathrm{C} \equiv \mathrm{N}), 1639(\mathrm{C}=\mathrm{O}), 1355-$ $1315\left(\mathrm{NO}_{2}\right), 774(\mathrm{C}-\mathrm{Cl}) ; \mathrm{MS} \mathrm{m} / \mathrm{z}(\%): 425\left(\mathrm{M}^{+}\right), 304(25)$, 288 (100), 237 (15), 210 (12), 187 (5), 152 (7), 126 (13), Elemental Analysis for $\mathrm{C}_{19} \mathrm{H}_{9} \mathrm{Cl}_{2} \mathrm{~N}_{5} \mathrm{O}_{3}$ : Calculated: C, 53.54; H, 2.13; N, 16.43. Found: C, 53.22; H, 2.04; N, 16.18\%.

6-Amino-1-(2,5-dichlorophenyl)-4-(2-nitrophenyl)-2oxo-1,2-dihydro pyridine-3,5-dicarbonitrile (CP-5):

MP: $234-236^{\circ} \mathrm{C}$; ${ }^{1} \mathrm{H}-\mathrm{NMR}\left(400 \mathrm{MHz}\right.$, DMSO- $\left.d_{6}\right) \delta \mathrm{ppm}$ : $8.10-8.13\left(\mathrm{~d}, 2 \mathrm{H}, 2-\mathrm{NO}_{2}-\mathrm{Ar}-\mathrm{H}, J=11.6 \mathrm{~Hz}\right), 7.22-7.25$ (d, 2H, 2- $\mathrm{NO}_{2}-\mathrm{Ar}-\mathrm{H}, J=11.6 \mathrm{~Hz}$ ), 7.54-7.57 (t, 2H, 2$\left.\mathrm{NO}_{2}-\mathrm{Ar}-\mathrm{H}\right), 7.65-7.67$ (d, 1H, diCl-Ar-H), 7.71-7.74 (d, 1H, diCl-Ar-H), 7.84 (s, 1H, diCl-Ar-H), 8.28 (s, 2H, $\left.\mathrm{NH}_{2}\right)$; IR $\left(\mathrm{KBr}, \mathrm{v} / \mathrm{cm}^{-1}\right)$ : 3665 and $3522\left(\mathrm{NH}_{2}\right), 3194$ ( $\mathrm{Ar}-\mathrm{H}), 2218(\mathrm{C} \equiv \mathrm{N}), 1639(\mathrm{C}=\mathrm{O}), 1355-1315\left(\mathrm{NO}_{2}\right), 774$ (C-Cl); MS m/z (\%): 425(M ), 304 (25), 288 (100), 237 (15), 210 (12), 187 (5), 152 (7), 126 (13). Elemental Analysis for $\mathrm{C}_{19} \mathrm{H}_{9} \mathrm{Cl}_{2} \mathrm{~N}_{5} \mathrm{O}_{3}$ : Calculated: $\mathrm{C}, 53.54 ; \mathrm{H}, 2.13 ; \mathrm{N}$, 16.43. Found: C, 53.20; H, 2.08; N, 16.26\%.

6-Amino-1-(2,5-dichlorophenyl)-2-oxo-4-(p-tolyl)-1,2dihydro pyridine-3,5-dicarbonitrile (CP-6):

MP: $284-286{ }^{\circ} \mathrm{C} ;{ }^{1} \mathrm{H}-\mathrm{NMR}\left(400 \mathrm{MHz}, \mathrm{DMSO}-d_{6}\right) \delta \mathrm{ppm}$ : $2.05\left(\mathrm{~s}, 3 \mathrm{H}, \mathrm{CH}_{3}\right), 7.10-7.13\left(\mathrm{~d}, 2 \mathrm{H}, \mathrm{CH}_{3}-\mathrm{Ar}-\mathrm{H}, J=11.6\right.$ $\mathrm{Hz}), 7.42-7.45\left(\mathrm{~d}, 2 \mathrm{H}, \mathrm{OCH}_{3}-\mathrm{Ar}-\mathrm{H}, J=11.6 \mathrm{~Hz}\right), 7.68-$ 7.69 (d, 1H, diCl-Ar-H), 7.75-7.78 (d, 1H, diCl-Ar-H), 7.85 (s, $1 \mathrm{H}, \mathrm{diCl}-\mathrm{Ar}-\mathrm{H}), 8.28\left(\mathrm{~s}, 2 \mathrm{H}, \mathrm{NH}_{2}\right) ; \mathrm{IR}\left(\mathrm{KBr}, \mathrm{u} / \mathrm{cm}^{-1}\right)$ : 3665 and $3522\left(\mathrm{NH}_{2}\right), 2870\left(\mathrm{CH}_{3}\right), 3194(\mathrm{Ar}-\mathrm{C}-\mathrm{H}), 2218$ $(\mathrm{C} \equiv \mathrm{N}), 1639(\mathrm{C}=\mathrm{O}), 774(\mathrm{C}-\mathrm{Cl}) ; \mathrm{MS} \mathrm{m} / \mathrm{z}(\%): 394\left(\mathrm{M}^{+}\right)$, 380 (10), 359 (95), 282 (5), 254 (8), 233 (9), 202 (12), 142 (18), 91 (13), 77 (27). Elemental Analysis for $\mathrm{C}_{20} \mathrm{H}_{12} \mathrm{Cl}_{2} \mathrm{~N}_{4} \mathrm{O}$ : Calculated: C, 60.78; H, 3.06; N, 14.18. Found: C, 60.68; $\mathrm{H}, 3.00 ; \mathrm{N}, 14.10 \%$.
6-Amino-1-(2,5-dichlorophenyl)-4-(4-bromophenyl)2-oxo-1,2-dihydro pyridine-3,5-dicarbonitrile (CP-7): MP: $298-300{ }^{\circ} \mathrm{C} ;{ }^{1} \mathrm{H}-\mathrm{NMR}\left(400 \mathrm{MHz}, \mathrm{DMSO}-d_{6}\right) \delta \mathrm{ppm}$ : $7.53-7.56$ (d, 2H, 4-Br-Ar-H, $J=11.6 \mathrm{~Hz}$ ), 7.79-7.82 (d, $2 \mathrm{H}, 4-\mathrm{Br}-\mathrm{Ar}-\mathrm{H}, J=11.6 \mathrm{~Hz}), 7.68-7.69(\mathrm{~d}, 1 \mathrm{H}, \mathrm{diCl}-\mathrm{Ar}-\mathrm{H})$, 7.75-7.78 (d, 1H, diCl-Ar-H), 7.85 (s, 1H, diCl-Ar-H), 8.28 (s, $\left.2 \mathrm{H}, \mathrm{NH}_{2}\right)$; IR $\left(\mathrm{KBr}, \mathrm{v} / \mathrm{cm}^{-1}\right)$ : 3665 and $3522\left(\mathrm{NH}_{2}\right)$, $3194(\mathrm{Ar}-\mathrm{H}), 2218(\mathrm{C} \equiv \mathrm{N}), 1639(\mathrm{C}=\mathrm{O}), 774(\mathrm{C}-\mathrm{Cl}), 642$ (C-Br); MS m/z (\%): $460\left(\mathrm{M}^{+}\right), 425$ (95), 401 (23), 323 (18), 293 (10), 205 (12), 218 (8), 164 (9), 77 (65). Elemental Analysis for $\mathrm{C}_{19} \mathrm{H}_{9} \mathrm{BrCl}_{2} \mathrm{~N}_{4} \mathrm{O}$ : Calculated: $\mathrm{C}, 49.60 ; \mathrm{H}, 1.97 ; \mathrm{N}$, 12.18. Found: C, 49.37; H, 1.85; N, 12.07\%.

6-Amino-1-(2,5-dichlorophenyl)-4-(3-bromophenyl)2-oxo-1,2-dihydro pyridine-3,5-dicarbonitrile (CP-8): MP: $305-308^{\circ} \mathrm{C} ;{ }^{1} \mathrm{H}-\mathrm{NMR}\left(400 \mathrm{MHz}\right.$, DMSO- $\left.d_{6}\right) \delta \mathrm{ppm}$ : 7.38-7.41 (m, 3H, 3-Cl-Ar-H), 7.49 (s, 1H, 3-Cl-Ar-H), 7.68-7.69 (d, 1H, diCl-Ar-H), 7.75-7.78 (d, 1H, diCl$\mathrm{Ar}-\mathrm{H}), 7.85$ (s, 1H, diCl-Ar-H), 8.28 (s, 2H, NH $\mathrm{N}_{2}$ ) IR $\left(\mathrm{KBr}, \mathrm{v} / \mathrm{cm}^{-1}\right): 3665$ and $3522\left(\mathrm{NH}_{2}\right), 3194(\mathrm{Ar}-\mathrm{C}-\mathrm{H}), 2218$ $(\mathrm{C} \equiv \mathrm{N}), 1639(\mathrm{C}=\mathrm{O}), 774(\mathrm{C}-\mathrm{Cl}), 642(\mathrm{C}-\mathrm{Br}) ; \mathrm{MS} \mathrm{m} / \mathrm{z}(\%)$ : $460\left(\mathrm{M}^{+}\right), 425$ (95), 401 (23), 323 (18), 293 (10), 205 (12), 218 (8), 164 (9), 77 (65). Elemental Analysis for $\mathrm{C}_{19} \mathrm{H}_{9} \mathrm{BrCl}_{2} \mathrm{~N}_{4} \mathrm{O}$ : Calculated: C, 49.60; H, 1.97; N, 12.18. Found: C, 49.27; $\mathrm{H}, 1.90 ; \mathrm{N}, 12.11 \%$.

6-Amino-1-(2,5-dichlorophenyl)-4-(4-chlorophenyl)2-oxo-1,2-dihydro pyridine-3,5-dicarbonitrile (CP-9): MP: $278-282^{\circ} \mathrm{C} ;{ }^{1} \mathrm{H}-\mathrm{NMR}\left(400 \mathrm{MHz}\right.$, DMSO- $\left.d_{6}\right) \delta \mathrm{ppm}$ : $7.50-7.53(\mathrm{~d}, 2 \mathrm{H}, 4-\mathrm{Cl}-\mathrm{Ar}-\mathrm{H}, J=11.6 \mathrm{~Hz}), 7.75-7.78(\mathrm{~d}$, $2 \mathrm{H}, 4-\mathrm{Cl}-\mathrm{Ar}-\mathrm{H}, J=11.6 \mathrm{~Hz}), 7.68-7.69(\mathrm{~d}, 1 \mathrm{H}, \mathrm{diCl}-\mathrm{Ar}-\mathrm{H})$, 7.75-7.78 (d, 1H, diCl-Ar-H), 7.85 (s, 1H, diCl-Ar-H), 8.28 (s, $\left.2 \mathrm{H}, \mathrm{NH}_{2}\right)$; IR $\left(\mathrm{KBr}, \mathrm{v} / \mathrm{cm}^{-1}\right)$ : 3665 and $3522\left(\mathrm{NH}_{2}\right)$, $3194(\mathrm{Ar}-\mathrm{H}), 2218(\mathrm{C} \equiv \mathrm{N}), 1639(\mathrm{C}=\mathrm{O}), 774(\mathrm{C}-\mathrm{Cl})$; MS $\mathrm{m} / \mathrm{z}(\%): 414\left(\mathrm{M}^{+}\right), 379(90), 304$ (21), 288 (25), 254 (5), 210 (15), 142 (8), 77 (29). Elemental Analysis for $\mathrm{C}_{19} \mathrm{H}_{9} \mathrm{Cl}_{3} \mathrm{~N}_{4} \mathrm{O}$ : Calculated: C, 54.90; H, 2.18 N, 13.48. Found: C, 54.70; $\mathrm{H}, 2.10 \mathrm{~N}, 13.25 \%$.

6-Amino-1-(2,5-dichlorophenyl)-4-(3-chlorophenyl)2-oxo-1,2-dihydro pyridine-3,5-dicarbonitrile (CP-10): MP: $285-288^{\circ} \mathrm{C} ;{ }^{1} \mathrm{H}-\mathrm{NMR}\left(400 \mathrm{MHz}, \mathrm{DMSO}-d_{6}\right) \delta \mathrm{ppm}$ : 7.28-7.31 (m, 3H, 3-Cl-Ar-H), 7.45 (s, 1H, 3-Cl-Ar-H), 7.68-7.69 (d, 1H, diCl-Ar-H), 7.75-7.78 (d, 1H, diCl-Ar-H), $7.85(\mathrm{~s}, 1 \mathrm{H}, \mathrm{diCl}-\mathrm{Ar}-\mathrm{H}), 8.28\left(\mathrm{~s}, 2 \mathrm{H}, \mathrm{NH}_{2}\right) ; \mathrm{IR}\left(\mathrm{KBr}, \mathrm{v} / \mathrm{cm}^{-1}\right)$ : 3665 and $3522\left(\mathrm{NH}_{2}\right), 3194(\mathrm{Ar}-\mathrm{H}), 2218(\mathrm{C} \equiv \mathrm{N}), 1639$ $(\mathrm{C}=\mathrm{O}), 774(\mathrm{C}-\mathrm{Cl}) ; \mathrm{MS} \mathrm{m} / \mathrm{z}(\%)$ : $414\left(\mathrm{M}^{+}\right), 379$ (90), 304 (21), 288 (25), 254 (5), 210 (15), 142 (8), 77 (29). Elemental Analysis for $\mathrm{C}_{19} \mathrm{H}_{9} \mathrm{Cl}_{3} \mathrm{~N}_{4} \mathrm{O}$ : Calculated: $\mathrm{C}, 54.90 ; \mathrm{H}, 2.18$ $\mathrm{N}, 13.48$. Found: C, $54.55 ; \mathrm{H}, 2.08 \mathrm{~N}, 13.14 \%$.

6-Amino-1-(2,5-dimethylphenyl)-2-oxo-4-phenyl-1,2dihydro pyridine-3,5-dicarbonitrile (CP-11): 
MP: $283-285^{\circ} \mathrm{C} ;{ }^{1} \mathrm{H}-\mathrm{NMR}\left(400 \mathrm{MHz}, \mathrm{DMSO}-d_{6}\right) \delta \mathrm{ppm}$ : $2.02\left(\mathrm{~s}, 3 \mathrm{H}, \mathrm{CH}_{3}\right), 2.32\left(\mathrm{~s}, 3 \mathrm{H}, \mathrm{CH}_{3}\right), 7.55$ (s, 5H, Ar-H), 7.25-7.35 (d, 2H, diCH $3-\mathrm{Ar}-\mathrm{H}), 7.12-7.15$ (d, 2H, 4$\left.\mathrm{OCH}_{3}-\mathrm{Ar}-\mathrm{H}, J=11.6 \mathrm{~Hz}\right), 7.52-7.55$ (d, 2H, 4-OCH $3-\mathrm{Ar}-$ $\mathrm{H}, J=11.6 \mathrm{~Hz}), 7.10$ (s, 1H, diCH $3-\mathrm{Ar}-\mathrm{H}), 7.90$ (s, 2H, $\left.\mathrm{NH}_{2}\right)$, IR $\left(\mathrm{KBr}, \mathrm{v} / \mathrm{cm}^{-1}\right): 3641$ and $3447(\mathrm{~N}-\mathrm{H}), 3206$ $(\mathrm{Ar}-\mathrm{H}), 2870\left(\mathrm{CH}_{3}\right), 2215(\mathrm{C} \equiv \mathrm{N}), 1631(\mathrm{C}=\mathrm{O}), 774(\mathrm{C}-$ $\mathrm{Cl}) ; \mathrm{MS} \mathrm{m} / \mathrm{z}(\%): 340\left(\mathrm{M}^{+}, 24\right), 325$ (100), 309 (20), 164 (24), 107 (18), 103 (28), 91 (45), 77 (36). Elemental Analysis for $\mathrm{C}_{21} \mathrm{H}_{16} \mathrm{~N}_{4} \mathrm{O}$ : Calculated: $\mathrm{C}, 74.10 ; \mathrm{H}, 4.74 ; \mathrm{N}$, 16.46. Found: C, 74.06; H, 4.64; N, 16.56\%.

6-Amino-1-(2,5-dimethylphenyl)-4-(4-methoxyphenyl)2-oxo-1,2-dihydro pyridine-3,5-dicarbonitrile (CP-12): MP: $255-260{ }^{\circ} \mathrm{C} ;{ }^{1} \mathrm{H}-\mathrm{NMR}\left(400 \mathrm{MHz}, \mathrm{DMSO}-d_{6}\right) \delta \mathrm{ppm}$ : $2.02\left(\mathrm{~s}, 3 \mathrm{H}, \mathrm{CH}_{3}\right), 2.32\left(\mathrm{~s}, 3 \mathrm{H}, \mathrm{CH}_{3}\right), 3.85\left(\mathrm{~s}, 3 \mathrm{H}, \mathrm{OCH}_{3}\right)$, 7.25-7.35 (d, 2H, diCH $3-\mathrm{Ar}-\mathrm{H}), 7.12-7.15\left(\mathrm{~d}, 2 \mathrm{H}, 4-\mathrm{OCH}_{3}-\right.$ $\mathrm{Ar}-\mathrm{H}, J=11.6 \mathrm{~Hz}), 7.52-7.55\left(\mathrm{~d}, 2 \mathrm{H}, 4-\mathrm{OCH}_{3}-\mathrm{Ar}-\mathrm{H}, J\right.$ $=11.6 \mathrm{~Hz}), 7.10\left(\mathrm{~s}, 1 \mathrm{H}, \mathrm{diCH}_{3}-\mathrm{Ar}-\mathrm{H}\right), 7.90\left(\mathrm{~s}, 2 \mathrm{H}, \mathrm{NH}_{2}\right)$; IR $\left(\mathrm{KBr}, \mathrm{v} / \mathrm{cm}^{-1}\right): 3651$ and $3457(\mathrm{~N}-\mathrm{H}), 3206(\mathrm{Ar}-\mathrm{H})$, $2870\left(\mathrm{CH}_{3}\right), 2215(\mathrm{C} \equiv \mathrm{N}), 1631(\mathrm{C}=\mathrm{O}), 1172(\mathrm{C}-\mathrm{O}-\mathrm{C}$ symmetrical stretching $\mathrm{OCH}_{3}$ group); MS m/z (\%): 370(M+, 20), 355 (95), 324 (17), 205 (20), 164 (17), 107 (15), 103 (24), 91 (40), 77 (30). Elemental Analysis for $\mathrm{C}_{22} \mathrm{H}_{18} \mathrm{~N}_{4} \mathrm{O}_{2}$ : Calculated: C, 71.34; H, 4.90; N, 15.13.Found: C, 71.30; H, 4.87; N, $15.08 \%$.

6-Amino-1-(2,5-dimethylphenyl)-4-(2-methoxyphenyl)2-oxo-1,2-dihydro pyridine-3,5-dicarbonitrile (CP-13): MP: $225-228^{\circ} \mathrm{C} ;{ }^{1} \mathrm{H}-\mathrm{NMR}\left(400 \mathrm{MHz}, \mathrm{DMSO}-d_{6}\right) \delta \mathrm{ppm}$ : $2.02\left(\mathrm{~s}, 3 \mathrm{H}, \mathrm{CH}_{3}\right), 2.32\left(\mathrm{~s}, 3 \mathrm{H}, \mathrm{CH}_{3}\right), 3.85\left(\mathrm{~s}, 3 \mathrm{H}, \mathrm{OCH}_{3}\right)$, 7.10-7.13 (d, 2H, 4-OCH $-\mathrm{Ar}-\mathrm{H}, J=11.6 \mathrm{~Hz}), 7.54-7.57$ (d, 2H, 4-OCH $3-\mathrm{Ar}-\mathrm{H}, J=11.6 \mathrm{~Hz}$ ), 7.22-7.28 (t, 2H, 4$\left.\mathrm{OCH}_{3}-\mathrm{Ar}-\mathrm{H}\right), 7.25-7.35$ (d, 2H, diCH $\left.3-\mathrm{Ar}-\mathrm{H}\right), 7.10$ (s, $1 \mathrm{H}$, $\left.\mathrm{diCH}_{3}-\mathrm{Ar}-\mathrm{H}\right), 7.90$ (s, 2H, $\left.\mathrm{NH}_{2}\right)$; IR $\left(\mathrm{KBr}, \mathrm{v} / \mathrm{cm}^{-1}\right): 3650$ and $3440(\mathrm{~N}-\mathrm{H}), 3206(\mathrm{C}-\mathrm{H}$ aromatic $), 2870\left(\mathrm{CH}_{3}\right), 2215$ $(\mathrm{C} \equiv \mathrm{N}), 1631(\mathrm{C}=\mathrm{O}), 1172(\mathrm{C}-\mathrm{O}-\mathrm{C}$ symmetrical stretching $\mathrm{OCH}_{3}$ group); $\mathrm{MS} \mathrm{m} / \mathrm{z}(\%): 370\left(\mathrm{M}^{+}, 22\right), 355$ (96), 324 (14), 205 (19), 164 (5), 107 (10), 103 (32), 91 (30), 77 (35). Elemental Analysis for $\mathrm{C}_{22} \mathrm{H}_{18} \mathrm{~N}_{4} \mathrm{O}_{2}$ : Calculated: $\mathrm{C}, 71.34 ; \mathrm{H}$, 4.90; N, 15.13. Found: C, 71.31; H, 4.85; N, 15.10\%.

6-Amino-1-(2,5-dimethylphenyl)-4-(4-nitrophenyl)2-oxo-1,2-dihydro pyridine-3,5-dicarbonitrile (CP-14): MP: $195-198^{\circ} \mathrm{C} ;{ }^{1} \mathrm{H}-\mathrm{NMR}\left(400 \mathrm{MHz}, \mathrm{DMSO}-d_{6}\right) \delta \mathrm{ppm}$ : $2.02\left(\mathrm{~s}, 3 \mathrm{H}, \mathrm{CH}_{3}\right), 2.32\left(\mathrm{~s}, 3 \mathrm{H}, \mathrm{CH}_{3}\right), 7.11\left(\mathrm{~s}, 1 \mathrm{H}, \mathrm{diCH}_{3}-\mathrm{Ar}-\right.$ $\mathrm{H}), 7.25-7.35$ (d, 2H, diCH$\left.{ }_{3}-\mathrm{Ar}-\mathrm{H}\right), 7.58-7.61(\mathrm{~d}, 2 \mathrm{H}$, $4 \mathrm{Br}-\mathrm{Ar}-\mathrm{H}, J=11.2 \mathrm{~Hz}), 7.79-7.82(\mathrm{~d}, 2 \mathrm{H}, 4 \mathrm{Br}-\mathrm{Ar}-\mathrm{H}, J=$ $11.6 \mathrm{~Hz}), 7.91\left(\mathrm{~s}, 2 \mathrm{H}, \mathrm{NH}_{2}\right)$; IR $\left(\mathrm{KBr}, \mathrm{v} / \mathrm{cm}^{-1}\right)$ : 3644 and $3446(\mathrm{~N}-\mathrm{H}), 3200\left(\mathrm{C}-\mathrm{H}\right.$ aromatic), $2855\left(\mathrm{CH}_{3}\right), 2250(\mathrm{C} \equiv \mathrm{N})$, $1631(\mathrm{C}=\mathrm{O}), 1350-1310\left(\mathrm{NO}_{2}\right) ; \mathrm{MS} \mathrm{m} / \mathrm{z}(\%): 385\left(\mathrm{M}^{+}, 31\right)$, 370 (100), 339 (28), 205 (15), 164 (8), 122 (30), 103 (30),
77 (30). Elemental Analysis for $\mathrm{C}_{21} \mathrm{H}_{15} \mathrm{~N}_{5} \mathrm{O}_{3}$ : Calculated: C, 65.45; H, 3.92; N, 18.17.Found: C, 65.40; H, 3.91; N, $18.15 \%$.

6-Amino-1-(2,5-dimethylphenyl)-4-(2-nitrophenyl)2-oxo-1,2-dihydro pyridine-3,5-dicarbonitrile (CP-15): MP: $228-230{ }^{\circ} \mathrm{C} ;{ }^{1} \mathrm{H}-\mathrm{NMR}\left(400 \mathrm{MHz}, \mathrm{DMSO}-d_{6}\right) \delta \mathrm{ppm}$ : $1.90\left(\mathrm{~s}, 3 \mathrm{H}, \mathrm{CH}_{3}\right), 2.02$ (s, 3H, $\left.\mathrm{CH}_{3}\right), 7.25-7.35$ (d, 2H, $\left.\mathrm{diCH}_{3}-\mathrm{Ar}-\mathrm{H}\right), 7.10$ (s, 1H, diCH $\left.3-\mathrm{Ar}-\mathrm{H}\right), 7.90$ (s, 2H, $\mathrm{NH}_{2}$ ), 8.10-8.13 (d, 2H, 2-NO $\mathrm{NO}_{2}-\mathrm{Ar}-\mathrm{H}, J=11.6 \mathrm{~Hz}$ ), $7.22-7.25$ (d, 2H, 2-NO $2-\mathrm{Ar}-\mathrm{H}, J=11.6 \mathrm{~Hz}), 7.54-7.57$ (t, $\left.2 \mathrm{H}, 2-\mathrm{NO}_{2}-\mathrm{Ar}-\mathrm{H}\right)$; IR $\left(\mathrm{KBr}, \mathrm{v} / \mathrm{cm}^{-1}\right): 3351$ and $3457(\mathrm{~N}-$ $\mathrm{H}), 3206\left(\mathrm{C}-\mathrm{H}\right.$ aromatic), $2870\left(\mathrm{CH}_{3}\right), 2215(\mathrm{C} \equiv \mathrm{N}), 1631$ $(\mathrm{C}=\mathrm{O}), 1355-1315\left(\mathrm{NO}_{2}\right) ; \mathrm{MS} \mathrm{m} / \mathrm{z}(\%): 385\left(\mathrm{M}^{+}, 29\right), 370$ (98), 339 (30), 205 (14), 164 (6), 122 (21), 91 (8), 77 (45). Elemental Analysis for $\mathrm{C}_{21} \mathrm{H}_{15} \mathrm{~N}_{5} \mathrm{O}_{3}$ : Calculated: $\mathrm{C}, 65.45$; H, 3.92; N, 18.17. Found: C, 65.35; H, 3.88; N, 18.10\%.

6-Amino-1-(2,5-dimethylphenyl)-2-oxo-4-(p-tolyl)-1,2dihydro pyridine-3,5-dicarbonitrile (CP-16):

MP: $220-222{ }^{\circ} \mathrm{C} ;{ }^{1} \mathrm{H}-\mathrm{NMR}\left(400 \mathrm{MHz}, \mathrm{DMSO}-d_{6}\right) \delta \mathrm{ppm}$ : $1.90\left(\mathrm{~s}, 3 \mathrm{H}, \mathrm{CH}_{3}\right), 2.02\left(\mathrm{~s}, 3 \mathrm{H}, \mathrm{CH}_{3}\right), 2.32\left(\mathrm{~s}, 3 \mathrm{H}, \mathrm{CH}_{3}\right)$, 3.85 (s, 3H, $\left.\mathrm{OCH}_{3}\right), 7.25-7.35$ (d, 2H, diCH $\left.3-\mathrm{Ar}-\mathrm{H}\right)$, $7.12-7.15$ (d, 2H, 4-OCH $-\mathrm{Ar}-\mathrm{H}, J=11.6 \mathrm{~Hz}), 7.52-$ 7.55 (d, 2H, 4-OCH $-\mathrm{Ar}-\mathrm{H}, J=11.6 \mathrm{~Hz}), 7.10$ (s, 1H, $\left.\mathrm{diCH}_{3}-\mathrm{Ar}-\mathrm{H}\right), 7.90$ (s, 2H, NH$)_{2}$; IR (KBr, v/cm $\left.{ }^{-1}\right): 3650$ and $3450(\mathrm{~N}-\mathrm{H}), 3205\left(\mathrm{C}-\mathrm{H}\right.$ aromatic), $2868\left(\mathrm{CH}_{3}\right), 2340$ $(\mathrm{C} \equiv \mathrm{N}), 1629(\mathrm{C}=\mathrm{O}) ; \mathrm{MS} \mathrm{m} / \mathrm{z}(\%): 354\left(\mathrm{M}^{+}, 36\right), 339(92)$, 308 (26), 205 (13), 164 (11), 103 (22), 91 (10), 77 (42). Elemental Analysis for $\mathrm{C}_{22} \mathrm{H}_{18} \mathrm{~N}_{4} \mathrm{O}$ : Calculated: $\mathrm{C}, 74.56 ; \mathrm{H}$, 5.12; N, 15.81. Found: C, 74.50; H, 5.11; N, 15.71\%.

6-Amino-1-(2,5-dimethylphenyl)-4-(4-bromophenyl)2-oxo-1,2-dihydro pyridine-3,5-dicarbonitrile (CP-17):

MP: $275-279^{\circ} \mathrm{C} ;{ }^{1} \mathrm{H}-\mathrm{NMR}\left(400 \mathrm{MHz}, \mathrm{DMSO}-d_{6}\right) \delta \mathrm{ppm}$ : $2.02\left(\mathrm{~s}, 3 \mathrm{H}, \mathrm{CH}_{3}\right), 2.32\left(\mathrm{~s}, 3 \mathrm{H}, \mathrm{CH}_{3}\right), 7.11\left(\mathrm{~s}, 1 \mathrm{H}, \mathrm{diCH}_{3}-\right.$ $\mathrm{Ar}-\mathrm{H}), 7.25-7.35$ (d, 2H, diCH $3-\mathrm{Ar}-\mathrm{H}), 7.53-7.56$ (d, 2H, $4 \mathrm{Br}-\mathrm{Ar}-\mathrm{H} J=11.2 \mathrm{~Hz}$ ), 7.79-7.82 (d, 2H, 4Br-Ar-H, $J$ $=11.6 \mathrm{~Hz}), 7.93\left(\mathrm{~s}, 2 \mathrm{H}, \mathrm{NH}_{2}\right)$; IR $\left(\mathrm{KBr}, \mathrm{v} / \mathrm{cm}^{-1}\right): 3640$ and $3438(\mathrm{~N}-\mathrm{H}), 3206\left(\mathrm{C}-\mathrm{H}\right.$ aromatic), $2870\left(\mathrm{CH}_{3}\right), 2215(\mathrm{C} \equiv \mathrm{N})$, $1631(\mathrm{C}=\mathrm{O}), 642$ (C-Br); MS m/z (\%): $418\left(\mathrm{M}^{+}, 61\right), 403$ (100), 323 (18), 309 (10), 205 (12), 164 (14), 103 (32), 91 (20), 77 (65). Elemental Analysis for $\mathrm{C}_{21} \mathrm{H}_{15} \mathrm{BrN}_{4} \mathrm{O}$ : Calculated: C, 60.16; H, 3.61; N, 13.36. Found: C, 60.11; H, $3.51 ; \mathrm{N}, 13.31 \%$.

6-Amino-1-(2,5-dimethylphenyl)-4-(3-bromophenyl)2-oxo-1,2-dihydro pyridine-3,5-dicarbonitrile (CP-18): MP: $290-292{ }^{\circ} \mathrm{C}$; ${ }^{1} \mathrm{H}-\mathrm{NMR}$ (400 MHz, DMSO- $\left.d_{6}\right) \delta \mathrm{ppm}$ : $2.02\left(\mathrm{~s}, 3 \mathrm{H}, \mathrm{CH}_{3}\right), 2.32\left(\mathrm{~s}, 3 \mathrm{H}, \mathrm{CH}_{3}\right), 7.11\left(\mathrm{~s}, 1 \mathrm{H}, \mathrm{diCH}_{3}-\right.$ $\mathrm{Ar}-\mathrm{H}), 7.25-7.35$ (d, 2H, diCH $3-\mathrm{Ar}-\mathrm{H}), 7.42-7.55$ (m, $4 \mathrm{H}, 3 \mathrm{Br}-\mathrm{Ar}-\mathrm{H}), 7.93$ (s, 2H, NH${ }_{2}$ ); IR ( $\left.\mathrm{KBr}, \mathrm{v} / \mathrm{cm}^{-1}\right): 3635$ and $3420(\mathrm{~N}-\mathrm{H}), 3206(\mathrm{C}-\mathrm{H}$ aromatic $), 2872\left(\mathrm{CH}_{3}\right), 2215$ 
$(\mathrm{C} \equiv \mathrm{N}), 1631(\mathrm{C}=\mathrm{O}), 642(\mathrm{C}-\mathrm{Br}) ; \mathrm{MS} \mathrm{m} / \mathrm{z}(\%): 418\left(\mathrm{M}^{+}\right.$, 57), 403 (100), 323 (20), 309 (10), 205 (9), 164 (14), 103 (30), 91 (18), 77 (65). Elemental Analysis for $\mathrm{C}_{21} \mathrm{H}_{15} \mathrm{BrN}_{4} \mathrm{O}$ : Calculated: C, 60.16; H, 3.61; N, 13.36. Found: C, 60.13; H, 3.57; N, 13.31\%.

6-Amino-1-(2,5-dimethylphenyl)-4-(4-chlorophenyl)2-oxo-1,2-dihydro pyridine-3,5-dicarbonitrile (CP-19): MP: $235-238^{\circ} \mathrm{C} ;{ }^{1} \mathrm{H}-\mathrm{NMR}\left(400 \mathrm{MHz}, \mathrm{DMSO}-d_{6}\right) \delta \mathrm{ppm}$ : $2.02\left(\mathrm{~s}, 3 \mathrm{H}, \mathrm{CH}_{3}\right), 2.32\left(\mathrm{~s}, 3 \mathrm{H}, \mathrm{CH}_{3}\right), 7.11\left(\mathrm{~s}, 1 \mathrm{H}, \mathrm{diCH}_{3}-\right.$ $\mathrm{Ar}-\mathrm{H}$ ), 7.25-7.35 (d, 2H, diCH $3-\mathrm{Ar}-\mathrm{H}), 7.50-7.53$ (d, 2H, $4 \mathrm{Br}-\mathrm{Ar}-\mathrm{H}, J=11.2 \mathrm{~Hz}$ ), 7.77-7.80 (d, 2H, 4Br-Ar-H, $J$ $=11.6 \mathrm{~Hz}), 7.91\left(\mathrm{~s}, 2 \mathrm{H}, \mathrm{NH}_{2}\right) ; \mathrm{IR}\left(\mathrm{KBr}, \mathrm{v} / \mathrm{cm}^{-1}\right): 3635$ and $3440(\mathrm{~N}-\mathrm{H}), 3202\left(\mathrm{C}-\mathrm{H}\right.$ aromatic), $2870\left(\mathrm{CH}_{3}\right), 2211(\mathrm{C} \equiv \mathrm{N})$, $1641(\mathrm{C}=\mathrm{O}), 772(\mathrm{C}-\mathrm{Cl}) ; \mathrm{MS} \mathrm{m} / \mathrm{z}(\%): 374\left(\mathrm{M}^{+}, 52\right), 359$ (100), 276 (21), 309 (8), 205 (11), 164 (15), 103 (25), 91 (15), 77 (60). Elemental Analysis for $\mathrm{C}_{21} \mathrm{H}_{15} \mathrm{ClN}_{4} \mathrm{O}$ : Calculated: C, 67.29; H, 4.03; N, 14.95. Found: C, 67.25; H, 3.95; N, $14.89 \%$.

6-Amino-1-(2,5-dimethylphenyl)-4-(3-chlorophenyl)2-oxo-1,2-dihydro pyridine-3,5-dicarbonitrile (CP-20): MP: $258-260{ }^{\circ} \mathrm{C} ;{ }^{1} \mathrm{H}-\mathrm{NMR}\left(400 \mathrm{MHz}, \mathrm{DMSO}-d_{6}\right) \delta \mathrm{ppm}$ : $2.02\left(\mathrm{~s}, 3 \mathrm{H}, \mathrm{CH}_{3}\right), 2.32\left(\mathrm{~s}, 3 \mathrm{H}, \mathrm{CH}_{3}\right), 7.11\left(\mathrm{~s}, 1 \mathrm{H}, \mathrm{diCH}_{3}-\mathrm{Ar}-\right.$ $\mathrm{H}), 7.25-7.35$ (d, 2H, diCH $-\mathrm{Ar}-\mathrm{H}), 7.42-7.55$ (m, 4H, $3 \mathrm{Br}-\mathrm{Ar}-\mathrm{H}), 7.93$ (s, 2H, NH ); IR ( KBr, v/ $\left.\mathrm{cm}^{-1}\right)$ : 3590 and $3457(\mathrm{~N}-\mathrm{H}), 3200\left(\mathrm{C}-\mathrm{H}\right.$ aromatic), $2860\left(\mathrm{CH}_{3}\right), 2210$ $(\mathrm{C} \equiv \mathrm{N}), 1621(\mathrm{C}=\mathrm{O}), 774(\mathrm{C}-\mathrm{Cl}) ; \mathrm{MS} \mathrm{m} / \mathrm{z}(\%): 374\left(\mathrm{M}^{+}, 55\right)$, 359 (98), 276 (16), 309 (6), 205 (10), 164 (12), 103 (28), 91 (18), 77 (58). Elemental Analysis for $\mathrm{C}_{21} \mathrm{H}_{15} \mathrm{ClN}_{4} \mathrm{O}$ : Calculated: C, 67.29; H, 4.03; N, 14.95. Found: C, 67.25; H, $4.00 ; \mathrm{N}, 14.92 \%$.

\section{Antimicrobial Screening}

The synthesized compound were tested for their antibacterial and antifungal activity (MIC) in vitro by broth dilution method ${ }^{19-21}$ with two Gram-positive bacteria Staphylococcus aureus (S.a.) MTCC 96, Streptococcus pyogenes (S.p.) MTCC 443, two Gram-negative bacteria Escherichia coli (E.c.) MTCC 442, Pseudomonas aeruginosa (P.a.) MTCC 441 and three fungal strains Candida albicans (C.a.) MTCC 227, Aspergillus niger (A.n.) MTCC 282, Aspergillus clavatus (A.c.) MTCC 1323 taking ampicillin, chloramphenicol, ciprofloxacin, norfloxacin, nystatin, and griseofulvin as standard drugs.

Serial dilutions of the test compounds and reference drugs were prepared in Muellere-Hinton agar. Drugs (10 mg) were dissolved in dimethylsulfoxide (DMSO, $1 \mathrm{~mL}$ ). Further progressive dilutions with melted Muellere-Hinton agar were performed to obtain the required concentrations of $1.56,3.12,6.25,10,12.5,25,50,62.5,100,125$,
250, 500 and $1000 \mu \mathrm{g} \mathrm{mL}^{-1}$. The tubes were inoculated with $10^{8}$ cfu mL $\mathrm{mL}^{-1}$ (colony forming unit $\mathrm{mL}^{-1}$ ) and incubated at $37^{\circ} \mathrm{C}$ for $24 \mathrm{~h}$. The MIC was the lowest concentration of the tested compound that yields no visible growth (turbidity) on the plate. To ensure that the solvent had no effect on the bacterial growth, a control was performed with the test medium supplemented with DMSO at the same dilutions as used in the experiments and it was observed that DMSO had no effect on the microorganisms in the concentrations studied.

\section{RESULTS AND DISCUSSION}

The compound $\mathbf{1}$ and $\mathbf{2}$ reacted in a solvent free condition to provide the starting materials (3) in good yields for the synthesis of desired product (CP 1-20).

As shown in the reaction (Scheme 2) the synthesis of the target molecules CP 1-20 was carried out. The compound 3 was reacted with the various substituted 4 and 5 compounds to give different target molecules. All the molecules have been synthesized under the conventional method using methanol as a solvent and pipyridine as a catalyst.

The physical data like molecular formula, molecular weight, melting point, and percentage of yield of all the synthesized target molecules are shown in Table 1.

The purity of all the synthesized compound was checked by TLC (8:2:n-hexane:ethylacetate). The target molecules were conformed on the basis of ${ }^{1} \mathrm{H}-\mathrm{NMR}$, IR, Mass Spectrometry. The ${ }^{1} \mathrm{H}-\mathrm{NMR}$ spectra showed the broad peak in between 7.5-8.0 ppm which showed the presence of an $-\mathrm{NH}_{2}$ functional group. The aromatic ring protons and $J$ values were found to be in accordance with substitution pattern on phenyl ring. The sharp band near $2250 \mathrm{~cm}^{-1}$ in IR spectra indicates the presence of $-\mathrm{CN}$ group. Systematic fragmentation pattern was observed in mass spectral analysis for all the synthesized compounds. Molecular ion peak was observed in agreement with molecular weight of respective compound. All the synthesized target molecules showed screening in vitro studies on the selected microorganism and fungal species. Among the tested compounds CP-1, CP-2, CP-7 and CP-15 exhibited more antimicrobial activity against gram positive and gram negative bacteria and CP-9, CP-10 and CP-17 exhibited comparatively moderate anti fungal activity (Table 2).

\section{CONCLUSION}

In summary we have developed a simple, one pot method for the preparation of a series of 6-amino-1-(2,5-disub- 
Table 1. Physical data of all the synthesized compounds

\begin{tabular}{llllllc}
\hline Code & $\mathrm{R}_{1}, \mathrm{R}_{2}$ & $\mathrm{R}_{3}$ & \multicolumn{1}{c}{ M.F. } & M.W. (gm/mole) & M.P. $\left({ }^{\circ} \mathrm{C}\right.$ ) & \% of yield \\
\hline $\mathrm{CP}-1$ & $\mathrm{Cl}$ & $\mathrm{H}$ & $\mathrm{C}_{19} \mathrm{H}_{10} \mathrm{Cl}_{2} \mathrm{~N}_{4} \mathrm{O}$ & 380 & $272-274$ & 71 \\
$\mathrm{CP}-2$ & $\mathrm{Cl}$ & $4-\mathrm{OCH}_{3}$ & $\mathrm{C}_{20} \mathrm{H}_{12} \mathrm{Cl}_{2} \mathrm{~N}_{4} \mathrm{O}_{2}$ & 410 & $280-284$ & 66 \\
$\mathrm{CP}-3$ & $\mathrm{Cl}$ & $2-\mathrm{OCH}_{3}$ & $\mathrm{C}_{20} \mathrm{H}_{12} \mathrm{Cl}_{2} \mathrm{~N}_{4} \mathrm{O}_{2}$ & 410 & $254-258$ & 75 \\
$\mathrm{CP}-4$ & $\mathrm{Cl}$ & $4-\mathrm{NO}_{2}$ & $\mathrm{C}_{19} \mathrm{H}_{9} \mathrm{Cl}_{2} \mathrm{~N}_{5} \mathrm{O}_{3}$ & 425 & $245-250$ & 74 \\
$\mathrm{CP}-5$ & $\mathrm{Cl}$ & $2-\mathrm{NO}_{2}$ & $\mathrm{C}_{19} \mathrm{H}_{9} \mathrm{Cl}_{2} \mathrm{~N}_{5} \mathrm{O}_{3}$ & 425 & $234-236$ & 78 \\
$\mathrm{CP}-6$ & $\mathrm{Cl}$ & $4-\mathrm{CH}_{3}$ & $\mathrm{C}_{20} \mathrm{H}_{12} \mathrm{Cl}_{2} \mathrm{~N}_{4} \mathrm{O}$ & 394 & $284-286$ & 72 \\
$\mathrm{CP}-7$ & $\mathrm{Cl}$ & $4-\mathrm{Br}$ & $\mathrm{C}_{19} \mathrm{H}_{9} \mathrm{BrCl}_{2} \mathrm{~N}_{4} \mathrm{O}$ & 458 & $298-300$ & 65 \\
$\mathrm{CP}-8$ & $\mathrm{Cl}$ & $3-\mathrm{Br}$ & $\mathrm{C}_{19} \mathrm{H}_{9} \mathrm{BrCl}_{2} \mathrm{~N}_{4} \mathrm{O}$ & 458 & $305-308$ & 61 \\
$\mathrm{CP}-9$ & $\mathrm{Cl}$ & $4-\mathrm{Cl}$ & $\mathrm{C}_{19} \mathrm{H}_{9} \mathrm{Cl}_{3} \mathrm{~N}_{4} \mathrm{O}$ & 414 & $278-282$ & 70 \\
$\mathrm{CP}-10$ & $\mathrm{Cl}$ & $3-\mathrm{Cl}$ & $\mathrm{C}_{19} \mathrm{H}_{9} \mathrm{Cl}_{3} \mathrm{~N}_{4} \mathrm{O}$ & 414 & $285-288$ & 72 \\
$\mathrm{CP}-11$ & $\mathrm{CH}$ & $\mathrm{H}$ & $\mathrm{C}_{21} \mathrm{H}_{16} \mathrm{~N}_{4} \mathrm{O}$ & 340 & $283-285$ & 68 \\
$\mathrm{CP}-12$ & $\mathrm{CH}$ & $4-\mathrm{OCH}$ & $\mathrm{C}_{22} \mathrm{H}_{18} \mathrm{~N}_{4} \mathrm{O}_{2}$ & 370 & $255-260$ & 71 \\
$\mathrm{CP}-13$ & $\mathrm{CH}_{3}$ & $2-\mathrm{OCH}_{3}$ & $\mathrm{C}_{22} \mathrm{H}_{18} \mathrm{~N}_{4} \mathrm{O}_{2}$ & 370 & $225-228$ & 74 \\
$\mathrm{CP}-14$ & $\mathrm{CH}_{3}$ & $4-\mathrm{NO}_{2}$ & $\mathrm{C}_{21} \mathrm{H}_{15} \mathrm{~N}_{5} \mathrm{O}_{3}$ & 385 & $195-198$ & 65 \\
$\mathrm{CP}-15$ & $\mathrm{CH}_{3}$ & $2-\mathrm{NO}_{2}$ & $\mathrm{C}_{21} \mathrm{H}_{15} \mathrm{~N}_{5} \mathrm{O}_{3}$ & 385 & $228-230$ & 69 \\
$\mathrm{CP}-16$ & $\mathrm{CH}_{3}$ & $4-\mathrm{CH}_{3}$ & $\mathrm{C}_{22} \mathrm{H}_{18} \mathrm{~N}_{4} \mathrm{O}$ & 354 & $220-222$ & 74 \\
$\mathrm{CP}-17$ & $\mathrm{CH}_{3}$ & $4-\mathrm{Br}$ & $\mathrm{C}_{21} \mathrm{H}_{15} \mathrm{BrN}_{4} \mathrm{O}$ & 418 & $275-279$ & 70 \\
$\mathrm{CP}-18$ & $\mathrm{CH}_{3}$ & $3-\mathrm{Br}_{\mathrm{Cr}}$ & $\mathrm{C}_{21} \mathrm{H}_{15} \mathrm{BrN}_{4} \mathrm{O}$ & 418 & $290-292$ & 75 \\
$\mathrm{CP}-19$ & $\mathrm{CH}_{3}$ & $4-\mathrm{Cl}$ & $\mathrm{C}_{21} \mathrm{H}_{15} \mathrm{ClN}_{4} \mathrm{O}$ & 374 & $235-238$ & 68 \\
$\mathrm{CP}-20$ & $\mathrm{CH}_{3}$ & $3-\mathrm{Cl}$ & $\mathrm{C}_{21} \mathrm{H}_{15} \mathrm{ClN}_{4} \mathrm{O}$ & 374 & $258-260$ & 72 \\
\hline
\end{tabular}

Table 2. Minimal inhibitory concentration (MIC) of all synthesized compounds

\begin{tabular}{|c|c|c|c|c|c|c|c|}
\hline Code & S.a. & S.p. & E.c. & P.a. & C.a. & A.n. & A.c. \\
\hline$\overline{\mathrm{CP}}-1$ & 100 & 62.5 & 250 & 250 & $>1000$ & 500 & 100 \\
\hline CP-2 & 100 & 500 & 62.5 & 250 & 500 & $>1000$ & 1000 \\
\hline CP-3 & 200 & 250 & 200 & 100 & 500 & 1000 & 1000 \\
\hline CP-4 & 100 & 500 & 125 & 1000 & 500 & 1000 & 1000 \\
\hline CP-5 & 250 & 1000 & 250 & 500 & 500 & 250 & 250 \\
\hline CP-6 & 1000 & 500 & 1000 & 1000 & 500 & 500 & 1000 \\
\hline CP-7 & 100 & 62.5 & 125 & 100 & 500 & 500 & 500 \\
\hline CP-8 & 100 & 1000 & 250 & 1000 & 1000 & 500 & 1000 \\
\hline CP-9 & 250 & 250 & 100 & 500 & 100 & 500 & 500 \\
\hline CP-10 & 125 & 100 & 100 & 500 & 500 & 100 & 1000 \\
\hline CP-11 & 500 & 500 & 250 & 200 & $>1000$ & $>1000$ & $>1000$ \\
\hline CP-12 & 250 & 200 & 150 & 100 & 500 & 500 & 250 \\
\hline CP-13 & 200 & 500 & 250 & 500 & 250 & 500 & $>1000$ \\
\hline CP-14 & 100 & 500 & 250 & 200 & $>1000$ & $>1000$ & $>1000$ \\
\hline CP-15 & 250 & 250 & 62.5 & 250 & 1000 & 1000 & 500 \\
\hline CP-16 & 500 & 500 & 250 & 500 & 250 & $>1000$ & $>1000$ \\
\hline CP-17 & 100 & 500 & 250 & 200 & 500 & 100 & 250 \\
\hline CP-18 & 250 & 250 & 500 & 250 & 1000 & 500 & $>1000$ \\
\hline CP-19 & 200 & 250 & 500 & 500 & 500 & 250 & 500 \\
\hline CP-20 & 500 & 100 & 500 & 500 & $>1000$ & $>1000$ & $>1000$ \\
\hline Ampicillin & 250 & 100 & 100 & 100 & - & - & - \\
\hline Chloramphenicol & 50 & 50 & 50 & 50 & - & - & - \\
\hline Ciprofloxacin & 50 & 50 & 25 & 25 & - & - & - \\
\hline Nystatin & - & - & - & - & 100 & 100 & 100 \\
\hline Greseofulvin & - & - & - & - & 500 & 100 & 100 \\
\hline
\end{tabular}


stituted phenyl)-2-oxo-4-substituted phenyl-1,2-dihydropyridine-3,5-dicarbonitrile. All the synthesized compounds were characterized by FT-IR, ${ }^{1} \mathrm{H}-\mathrm{NMR}$, mass spectroscopy and elemental analysis. The compounds were subjected for different biological activities and all the synthesized compounds showed good to moderate antimicrobial activity, results of other activities are awaiting. Further efforts toward this end will be reported in due course.

Acknowledgments. Authors are thankful to KSKV Kachchh University and M M Science College to providing research support and facility. Authurs are also thankful to Dr V R Ram and Dr G A Baxi for their help in spectral analysis. And the publication cost of this paper was supported by the Korean Chemical Society.

\section{REFERENCES}

1. Choi, W.; Houpis, I. N.; Charchil, H. R. O.; Molina, A.; Lynch, J. E.; Volante, R. P.; Reider, P. J.; King, A. O. Tetrahedron 1995, 36, 4571.

2. Bhupathy, M.; Conlon, D. A.; Wells, K. M.; Wells, M.; Nolson, J. R.; Reider, P. J.; Rossen, K.; Sager, J. W.; Volante, R. P. Cyanoacetanilides Intermediates in Heterocyclic Synthesis. Part 1: A Facile Synthesis of Polysubstituted and Condensed Pyridones J. Heterocyclic Chem. 1995, 32, 1283.

3. Kappe, O. C.; Kappe, T. Synthesis of substituted 3-pyridine carbo nitriles with potential biological activity Monatshefte fur Chemie 1989, 120, 1095.

4. Saiki, A. Y. C.; Shen, L. L.; Chen, C. M.; Baranowski, J.; Lerner, C. G. DNA Cleavage Activities of Staphylococcus aureus Gyrase and Topoisomerase IV Stimulated by Quinolones and 2-Pyridones Antimicrob. Agents Chemother. 1999, 43, 1574.

5. Brickner, S. Regio and Selective Synthesis of 4,6-Disubstituted-2-pyridones. Chem. Ind. 1997, 131.

6. Cheney, I. W.; Yan, S.; Appleby, T.; Walker, H.; Vo, T.; Yao, N.; Hamatake, R.; Hong, Z.; Wu, J. Z. Bioorg. Med. Chem. Lett. 2007, 17, 1679.

7. Wendt, M. D.; et al. Bioorg. Med. Chem. Lett. 2007, 17, 3122.

8. (a) Hardie, D. G. Endocrinology 2003, 144, 5179. (b) Hardie, D. G.; Scott, J. W.; Pan, D. A.; Hudson, E. R. FEBS Lett. 2003, 546, 113. (c) Kahn, B. B.; Alquier, T.; Carling, D.;
Hardie, D. G. Cell Metab. 2005, 1, 15. (d) Hawley, S. A.; Boudeau, J.; Reid, J. L.; Mustard, K. J.; Udd, L.; Makela, T. P.; Alessi, D. R.; Hardie, D. G. J. Biol. 2003, 2, 28.

9. Li, Q.; Mitscher, L. A.; Shen, L. L. The 2-Pyridone Antibacterial Agents: Bacterial Topoisomerase Inhibitors Med. Res. Rev. 2000, 20, 231.

10. Gupta, A, K.; Plott, T. Ciclopirox: A Broad-Spectrum Antifungal with Antibacterial and Anti-inflammatory Properties. Int. J. Dermatol. 2004, 43, 3.

11. Collins, I.; et al. 3-Heteroaryl-2-pyridones: Benzodiazepine Site Ligands with Functional Selectivity for $\alpha 2 / \alpha 3$-Subtypes of Human GABAA Receptor-Ion Channels $J$. Med. Chem. 2002, 45, 1887.

12. Robert, N.; Verrier, C.; Hoarau, C.; Celanire, S.; Marsais, F. Solid-phase Synthesis of 3-Hydroxy-2-methylenealkanenitriles Using Polymer-Supported $\alpha$-Selenopropionitrile. ARKIVOC 2008, 7, 92.

13. Sollogoub, M.; Fox, K. R.; Powers, V. E. C.; Brown, T. First Synthesis of 1-Deazacytidine, the C-nucleoside Analogue of Cytidine Tetrahedron Lett. 2002, 43, 3121.

14. Parsons, S.; Winpenny, R. E. P. Structural Chemistry of Pyridonate Complexes of Late 3d-Metals Acc. Chem. Res. 1997, 30, 89.

15. Murray, T.; Zimmerman, S. 7-Amido-1,8-naphthyridines as Hydrogen Bonding Units for the Complexation of Guanine Derivatives: The Role of 2-Alkoxyl Groups in Decreasing Binding Affinity Tetrahedron Lett. 1995, 36, 7627.

16. Werbitzky, O.; Studer, P. Process for the Production of 2Chloro-5-chloromethyl-pyridine. U.S. Patent 6,022,974, Feb 8, 2008.

17. Murata, T.; Shimizu, K.; Narita, M.; Manganiello, V.; Tagawa, T. Characterization of Phosphodiesterase 3 in Human Malignant Melanoma Cell Line Anticancer Res. 2000, 22, 3171.

18. Ahmed Ali, F.; Samir, B.; Ramy, R.; Hassan Ali, E. Cyanoacetamide Derivatives as Synthons in Heterocyclic Synthesis Turk. J. Chem. 2008, 32, 259.

19. National Committee for Clinical and Laboratory Standards (NCCLS). Method for Dilution Antimicrobial Susceptibility Tests for Bacteria that Grow Aerobically Approved Standard, 4th ed. NCCLS, Villanova, Italy. 1997, Document M 100-S7. S100-S157.

20. Isenberg, D. H. Essential Procedure for Clinical Microbiology; American Society for Microbiology: Washington, 1998.

21. Zgoda, J. R.; Porter, J. R. Pharm. Biol. 2001, 39, 221-25. 